\title{
DISCOVERY OF TIME VARIATION OF THE INTENSITY OF MOLECULAR LINES IN IRC+10216 IN THE SUBMILLIMETER AND FAR-INFRARED DOMAINS
}

\author{
J. Cernicharo ${ }^{1}$, D. Teyssier ${ }^{2}$, G. Quintana-Lacaci ${ }^{1}$, F. Daniel ${ }^{3,4}$, M. Agúndez ${ }^{1}$, L. Velilla-Prieto ${ }^{1}$, \\ L. Decin ${ }^{5}$, M. Guélin ${ }^{6}$, P. Encrenaz ${ }^{7}$, P. García-Lario ${ }^{2}$, E. De Beck ${ }^{8}$, M. J. Barlow ${ }^{9}$, \\ M. A. T. Groenewegen ${ }^{10}$, D. Neufeld ${ }^{11}$, and J. Pearson ${ }^{12}$ \\ ${ }^{1}$ Group of Molecular Astrophysics, ICMM, CSIC, C/Sor Juana Inés de La Cruz N3, E-28049 Madrid, Spain \\ ${ }^{2}$ ESA, ESAC, P.O. Box 78, Villanueva de la Cañada, E-28691 Madrid, Spain \\ ${ }^{3}$ Univ. Grenoble Alpes, IPAG, F-38000 Grenoble, France \\ ${ }^{4}$ CNRS, IPAG, F-38000 Grenoble, France \\ ${ }^{5}$ Instituut voor Sterrenkunde, Katholieke Universiteit Leuven, Celestijnenlaan 200D, B-3001 Leuven, Belgium \\ ${ }^{6}$ Institut de Radioastronomie Millimétrique, 300 rue de la Piscine, F-38406 St-Martin d'Hères, France \\ ${ }^{7}$ LERMA, Observatoire de Paris, 61 Av. de l'Observatoire, F-75014 Paris, France \\ ${ }^{8}$ Department of Earth and Space Sciences, Chalmers University of Technology, Onsala Space Observatory, SE 43992 Onsala, Sweden \\ ${ }^{9}$ Department of Physics and Astronomy, University College London, Gower Street, London WC1E 6BT, UK \\ ${ }^{10}$ Koninklijke Sterrenwacht van België, Ringlaan 3, B-1180 Brussels, Belgium \\ ${ }^{11}$ Department of Physics and Astronomy, Johns Hopkins University, 3400 North Charles Street, Baltimore, MD 21218, USA \\ 12 Jet Propulsion Laboratory, California Institute of Technology, Pasadena, CA 91109, USA \\ Received 2014 October 7; accepted 2014 October 20; published 2014 November 10
}

\begin{abstract}
We report on the discovery of strong intensity variations in the high rotational lines of abundant molecular species toward the archetypical circumstellar envelope of IRC+10216. The observations have been carried out with the Heterodyne Instrument for the Far-Infrared (HIFI) instrument on board Herschel and with the IRAM30 m telescope. They cover several observing periods spreading over three years. The line intensity variations for molecules produced in the external layers of the envelope most likely result from time variations in the infrared pumping rates. We analyze the main implications this discovery has on the interpretation of molecular line emission in the envelopes of Mira-type stars. Radiative transfer calculations must take into account both the time variability of infrared pumping and the possible variation of the dust and gas temperatures with stellar phase in order to reproduce the observation of molecular lines at different epochs. The effect of gas temperature variations with stellar phase could be particularly important for lines produced in the innermost regions of the envelope. Each layer of the circumstellar envelope sees the stellar light radiation with a different lag time (phase). Our results show that this effect must be included in the models. The submillimeter and far infrared lines of asymptotic giant branch stars can no longer be considered as safe intensity calibrators.
\end{abstract}

Key words: astrochemistry - stars: AGB and post-AGB - stars: carbon - stars: individual (IRC, 10216)

\section{INTRODUCTION}

Half of the known interstellar molecular species are detected in IRC+10216, the envelope of the asymptotic giant branch (AGB) star CW Leo and one of the brightest infrared sources in the sky. CW Leo, at a estimated distance of $\simeq 130 \mathrm{pc}$ from the Sun, is a Mira variable star with a period of 630-670 days and an amplitude of $\simeq 1$ mag in the $K$ band (Menten et al. 2012, and references therein).

Due to its proximity, its large mass-loss rate and wealth of molecules, IRC+10216 has attracted many studies and is considered as the archetype of high mass-loss AGB stars. Yet, the formation of the dust and of molecules, some of which are fairly complex, and their dependence on the stellar state of evolution, from AGB to pre-PN, are key issues that are not yet fully understood. The Herschel ${ }^{13}$ satellite (Pilbratt et al. 2010) recently allowed much progress in this domain. The stellar atmosphere and the dust condensation zone are best probed by ro-vibrational lines and by high- $J$ pure rotational lines pertaining to the ground and vibrationally excited states. Those lines lie in the infrared and far infrared (FIR) for the most abundant species,

13 Herschel is an ESA space observatory with science instruments provided by European-led Principal Investigator consortia and with important participation from NASA. such as CO, HCN, and SiS (Fonfría et al. 2008; Cernicharo et al. 1996, 2011; Decin et al. 2010). In contrast, the outer cold layers of the envelope are best probed by low- $J$ rotational lines at millimeter and submillimeter wavelengths.

The determination of the physical conditions throughout the envelope can be performed by combining a large set of FIR, millimeter, and submillimeter data. However, and despite the fact that IR pumping is recognized to play a role in the excitation of molecular lines in evolved stars (Agúndez \& Cernicharo 2006 for $\mathrm{H}_{2} \mathrm{O}$; Deguchi \& Uyemura 1984 for $\mathrm{HC}_{5} \mathrm{~N}$; Daniel et al. 2012 for HNC; de Beck et al. 2012 for $\mathrm{CCH}$ ), most studies carried out so far assumed steady state, i.e., that the known periodic variations of the stellar IR flux do not modulate molecular line emission. This was partly justified by the lack of evidence of line intensity variations, outside a few cases of strong maser emission in O-rich AGBs for which maser line and IR continuum intensities are clearly correlated (Pardo et al. 2004; Nakashima \& Deguchi 2007). Cernicharo et al. (2000), for example, unsuccessfully searched for line intensity variations in their $2 \mathrm{~mm}$ spectral survey of IR+10216, which combines observations scattered over $12 \mathrm{yr}$ and mostly detects low- $J$ transitions from fairly abundant species and their isotopologues.

We present the results of a time monitoring of molecular line thermal emission in IRC+10216 taken between 480 and $1907 \mathrm{GHz}$ with the Herschel/Heterodyne Instrument for the 
Far-Infrared (HIFI) instrument over three years, a time interval longer than the light period of IRC +10216 and with the IRAM $30 \mathrm{~m}$ telescope in a selected sample of frequencies between 85 and $350 \mathrm{GHz}$.

We focus in this Letter on the study of $\mathrm{CCH}(N=1-0,3-2$, $4-3,6-5,7-6,8-7)$ and $\mathrm{HNC}(J=1-0,3-2,6-5,7-6,8-7)$. We present the first evidence for strong time variation of the intensity of those lines, as well as of high excitation lines of several other molecular species.

\section{OBSERVATIONS AND RESULTS}

A line survey of IRC+10216 was carried out on 2010 May 11-15 with HIFI (de Graauw et al. 2010) using all HIFI bands between 480 and $1907 \mathrm{GHz}$. These data have been previously presented by Cernicharo et al. (2010b).

A second set of data was taken during 2010 November to search for light hydrides in this source $\left(\mathrm{HCl}, \mathrm{HF}, \mathrm{PH}_{3}\right.$; Cernicharo et al. 2010a; Agúndez et al. 2011, 2014). A total of 66 frequency settings with $4+4 \mathrm{GHz}$ bandwidth (upper sideband and lower sideband) were acquired covering a large number of lines observed in both periods. Some frequency settings for that proposal were taken in 2011 May. Both data sets have an rms noise of 7-12 $\mathrm{mK}$ in $3 \mathrm{~km} \mathrm{~s}^{-1}$ wide spectral channels.

The comparison of the lines taken at six month intervals revealed surprisingly large intensity variations, as illustrated on Figure 1. These variations, which reach a factor of three in some cases, are well above any possible instrumental effect: pointing errors, gain calibration, sideband imbalance, baseline ripples, spurious ghost lines, etc. We found, in particular, the following.

1. More than $95 \%$ of the lines of $\mathrm{SiC}_{2}$ observed in both periods have exactly the same intensity.

2. Spectra with lines exhibiting an intensity variation also contain many other lines, detected with very good signalto-noise ratio $(>10)$, which do not show any intensity fluctuation.

3. CS, SiO, and SiS show a differential effect. Low- $J$ transitions have low line intensity changes. However, this variation is larger with large $J$ reaching a value $\simeq 25 \%$ for the three species (see the last two panels of Figure 1 for SiS).

4. The above variations are not directly related to the source extent, as they are observed both for $\mathrm{SiS}$, which is spatially constrained near the star, and to $\mathrm{CCH}$, which is only observed in the outer envelope. The $\mathrm{CCH} N=6-5$ through 8-7 lines show a factor of three of change in intensity, whereas two $\mathrm{SiC}_{2}$ lines, partly blended with the high $\mathrm{CCH}$ fine structure component, remain constant.

5. Water vapor shows a variation of $50 \%$. Following the models of Agúndez \& Cernicharo (2006), which include infrared pumping of $\mathrm{H}_{2} \mathrm{O}$, this result could had been expected.

6. HNC shows a systematic change of $20 \%-50 \%$ in the integrated intensity of all its lines observed with HIFI.

7. The CO lines observed at both epochs have low $J s$ and show no significant intensity variation. However, the $J=17-16$ line of ${ }^{13} \mathrm{CO}$, the only ${ }^{13} \mathrm{CO}$ line observed in both periods, appears to vary in intensity by $20 \%$.

8. HCN lines below $J=14-13$ have strong changes, ranging from $20 \%-50 \%$. HCN is a peculiar case, with several regimes in the excitation of its ro-vibrational levels. This makes the excitation diagram quite complex: for example, in the inner shells, the $v_{2}$ bending state has a population reaching as much as $30 \%$ of that of the ground
state(Cernicharo et al. 1996, 2011). The modeling of the observed HCN emission requires the inclusion of vibrational levels up to 12,000 K (Cernicharo et al. 1996, 2011, 2013).

Prompted by these unexpected results, we succeeded in extending by three more years our IRC+10216 study with Herschel. In total, seven observing runs extending from 2010 May to 2013 May were made with HIFI, using the same observing procedure in all runs. In addition, complementary lower-frequency observations were carried out with the IRAM $30 \mathrm{~m}$ telescope between 2012 and 2013. Note that the observing periods were similar but not identical on both instruments due to scheduling and weather constraints (we only kept data taken when the precipitable water atmospheric content was between 1 and $10 \mathrm{~mm}$ ). The system temperatures were then in the range 80-300 $\mathrm{K}$ and the pointing errors below $3^{\prime \prime}$.

In this work, we will focus on the lines of $\mathrm{CCH}$ and $\mathrm{HNC}$ throughout 2010-2013. The 2010 May data, taken during the first HIFI line survey, have already been analyzed for $\mathrm{CCH}$, $\mathrm{HNC}, \mathrm{CO}$, and $\mathrm{SiC}_{2}$ and published (Cernicharo et al. 2010a, 2010b; Müller et al. 2012; de Beck et al. 2012; Daniel et al. 2012). Full results for all detected molecular species, including data from other Herschel instruments, will be published in a forthcoming paper.

Figure 2 shows the $\mathrm{CCH}$ line profiles (composed of several hyperfine components $-h f s-$ ) observed at different epochs and Figure 3 shows those of HNC. For both molecules, even the lowest $N / J$ rotational transitions exhibit time variations in intensity and shape. The amplitude of the $\mathrm{CCH}$ line intensity variations changes with the rotational quantum number $N$, the higher $N$, the larger the amplitude (a factor of three for $N=6-5$ and about 7 for $N=7-6$ ). This is consistent with the expectation that collisional excitation is less efficient for the higher rotational levels of $\mathrm{CCH}$, a molecule only present in the outer envelope, making infrared pumping relatively more efficient. It is worth noting that the strongest $h f s$ component of $\mathrm{CCH} N=3-2$ (Figure 2 second panel from top) is partially blended with a line of $\mathrm{SiC}_{2}\left(12_{0,12}-11_{0,11}\right)$, which shows little or no intensity variation. The IRAM $30 \mathrm{~m}$ telescope observations show many other molecular lines with no significant intensity changes. Hence, the observed time fluctuations of the $N=$ $1-0,3-2$, the 4-3 lines of $\mathrm{CCH}$ and $J=1-0$, and 3-2 of HNC are not produced by calibration or pointing errors.

Besides being definitively real, the $\mathrm{CCH}$ and $\mathrm{HNC}$ line intensity variations correlate well with the periodic stellar light variations. Figure 4 shows the observed line intensities, as derived from Figures 2 and 3, atop the predicted stellar IR intensity (derived from Menten et al. 2012 for a period of 630 days) after applying shifts of 51 days and 59 days for HNC and $\mathrm{CCH}$, respectively. This shift is similar to that observed by Groenewegen et al. (2012) for the FIR dust continuum emission. Infrared light variations, by modulation of radiative pumping of the molecular levels, and, possibly in the innermost layers of the CSE, by modulating the dust grain and gas temperatures, clearly appear as the cause of the observed molecular line intensity variations. These effects must be definitively included in radiative transfer (RT) models.

\section{DISCUSSION}

The results described above require a detailed and careful revision of the interpretation and modeling performed up to now. Most of the RT models were aimed at deriving the population 

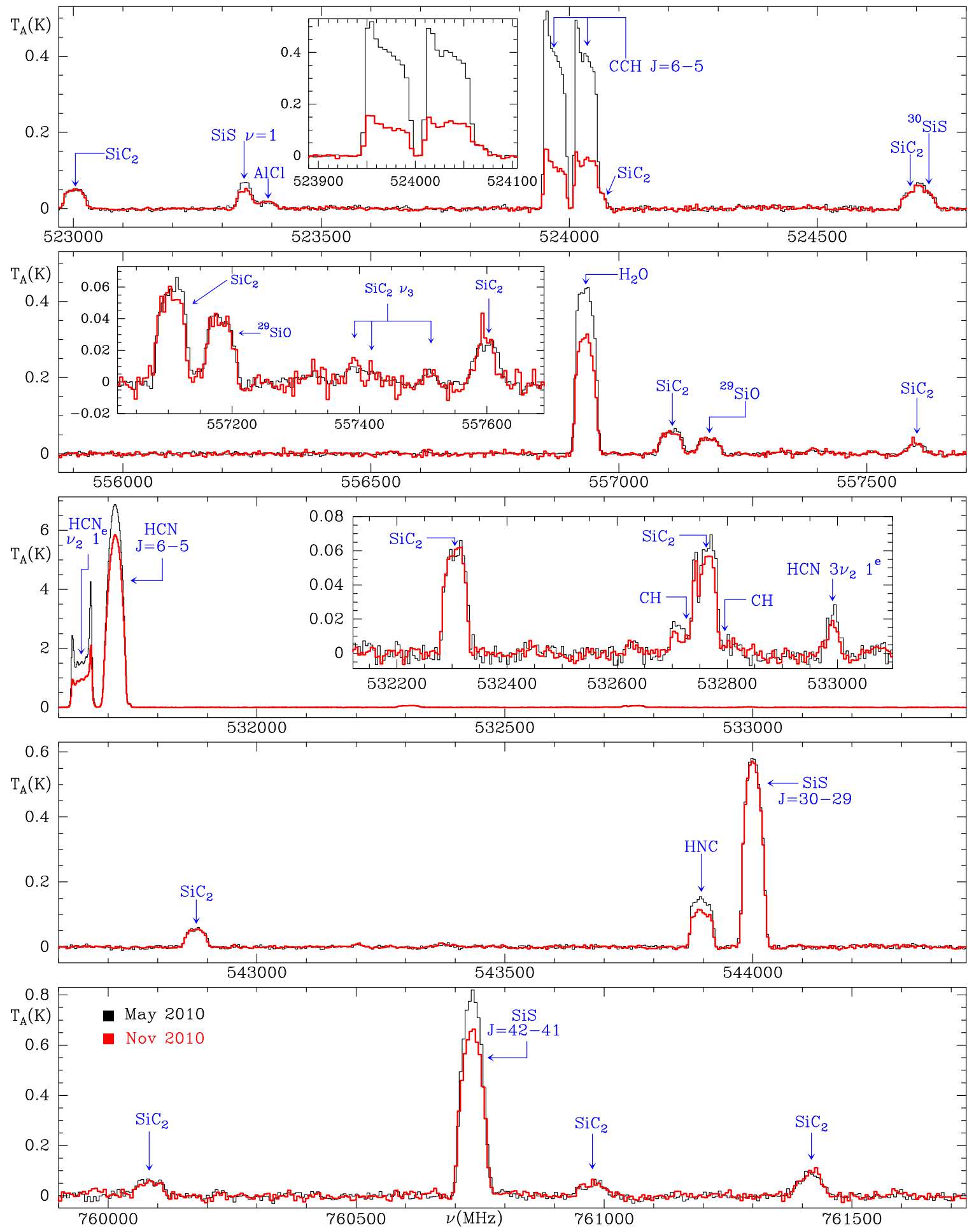

Figure 1. Selected spectra observed with Herschel in 2010 May (black thin spectra; from Cernicharo et al. 2010b) and 2010 November (red thick spectra). The spectrum at $543.5 \mathrm{GHz}$ was taken in 2011 May. Windows within the panels show zooms to selected lines. Intensity scale is antenna temperature in $\mathrm{K}$ and abscissa is frequency in MHz.

of the molecular energy levels of the abundant species, such as $\mathrm{CO}, \mathrm{HCN}, \mathrm{SiO}, \mathrm{SiS}, \mathrm{CS}, \mathrm{OH}$, and $\mathrm{H}_{2} \mathrm{O}$. All these molecules are characterized by thermal emission in their ground vibrational state. Nevertheless, some of them also show strong maser emission and these maser transitions can be used only to derive some estimates of the physical parameters of the envelope.

The modeling of the emission of thermal lines relies on assuming values, or taking them from observations when possible, for the temperatures and sizes for the central star and the dust formation zone (the latter may extend up to $20 R_{*}$; Fonfría et al. 2008), as well as the temperature and density profiles for the gas and the dust throughout the envelope. One generally assumes that the gas volume density varies as $n \simeq r^{-2}$ and that the geometry is spherically symmetric. One must also adopt an abundance profile for the key molecular species. The terminal expansion velocity in the envelope and the velocity field within 


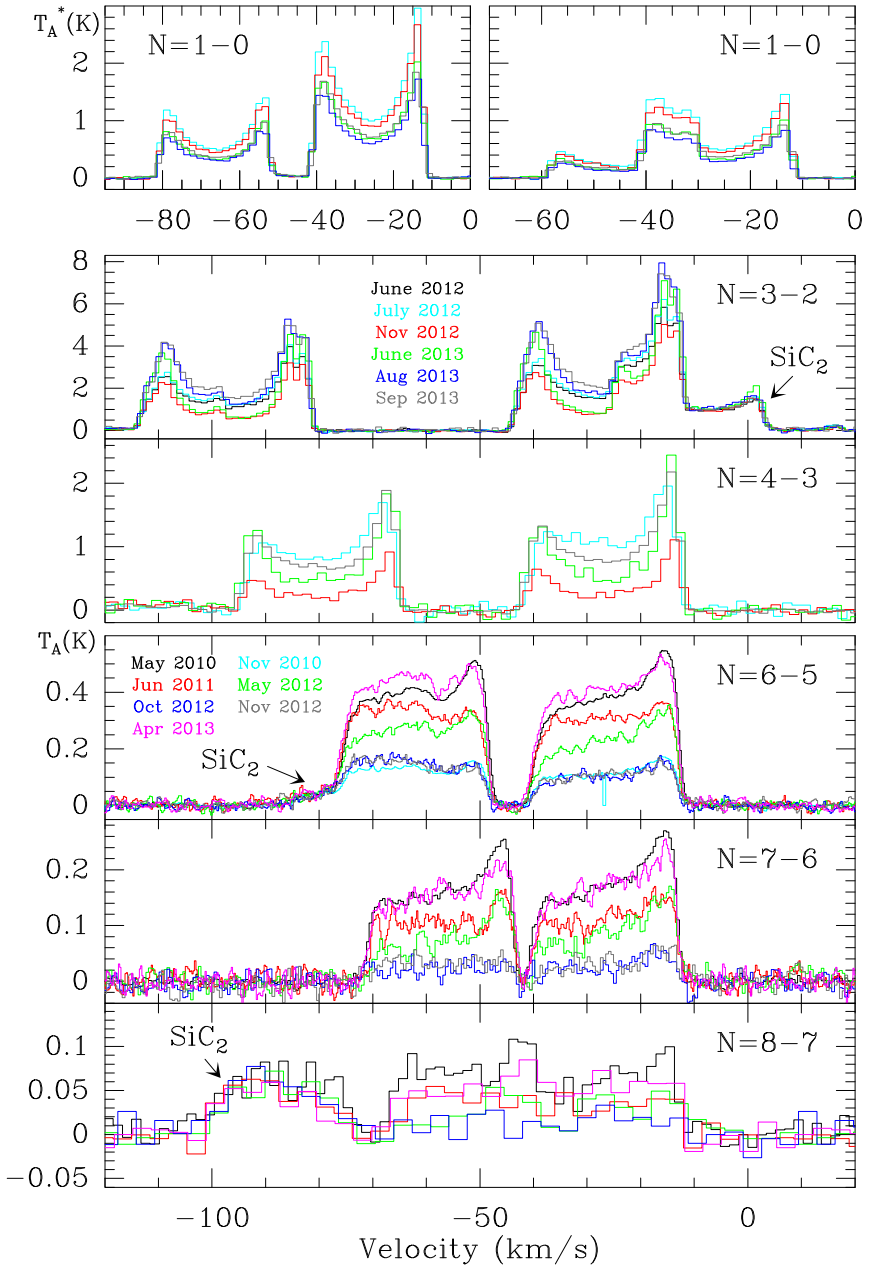

Figure 2. $\mathrm{CCH}$ lines observed with the IRAM $30 \mathrm{~m}$ telescope $(N=$ $1-0,3-2,4-3)$ and with HIFI $(N=6-5,7-6,8-7)$. The different colors correspond to the observing dates with both instruments. The abscissa is velocity in $\mathrm{km} \mathrm{s}^{-1}$ with respect the frequency of the strongest hyperfine component. Intensity scale is antenna temperature in $\mathrm{K}$.

the dust formation zone can then be derived from observations (see, e.g., Fonfría et al. 2008; Cernicharo et al. 2011).

From the observation of a large number of molecular lines of a given molecule with widely different energy levels several of these parameters can be constrained in steady state. However, when the central star and dust formation zone show significant flux intensity variations, i.e., when the steady state no longer holds, the problem becomes much more complex. The phase of the radiation field, seen at a given time by each point of the envelope, depends on the distance to the central radiation. Moreover, the temperature of the local gas, hence the collisional excitation rates, may also be modulated with different phase lags, since the gas heating and cooling are dominated in the inner regions by IR transitions that have large Einstein coefficients. The equilibrium times for the gas with the stellar and dust fluxes could there be fairly short. Statistical equilibrium equations assume that $d n_{i} / d t=0$, where $n_{i}$ represents the population of level $i$. However, now the source function, which describes the radiation field reaching a point of the envelope, depends on time in a complex way. Not only does the radiation directly incoming from the star and its surrounding hot dust shell come at a given point $\mathrm{P}$ of the envelope with a time lag, as described above, but each envelope point radiatively connected to $\mathrm{P}$ sees this stellar radiation with a different phase, because it lies at different

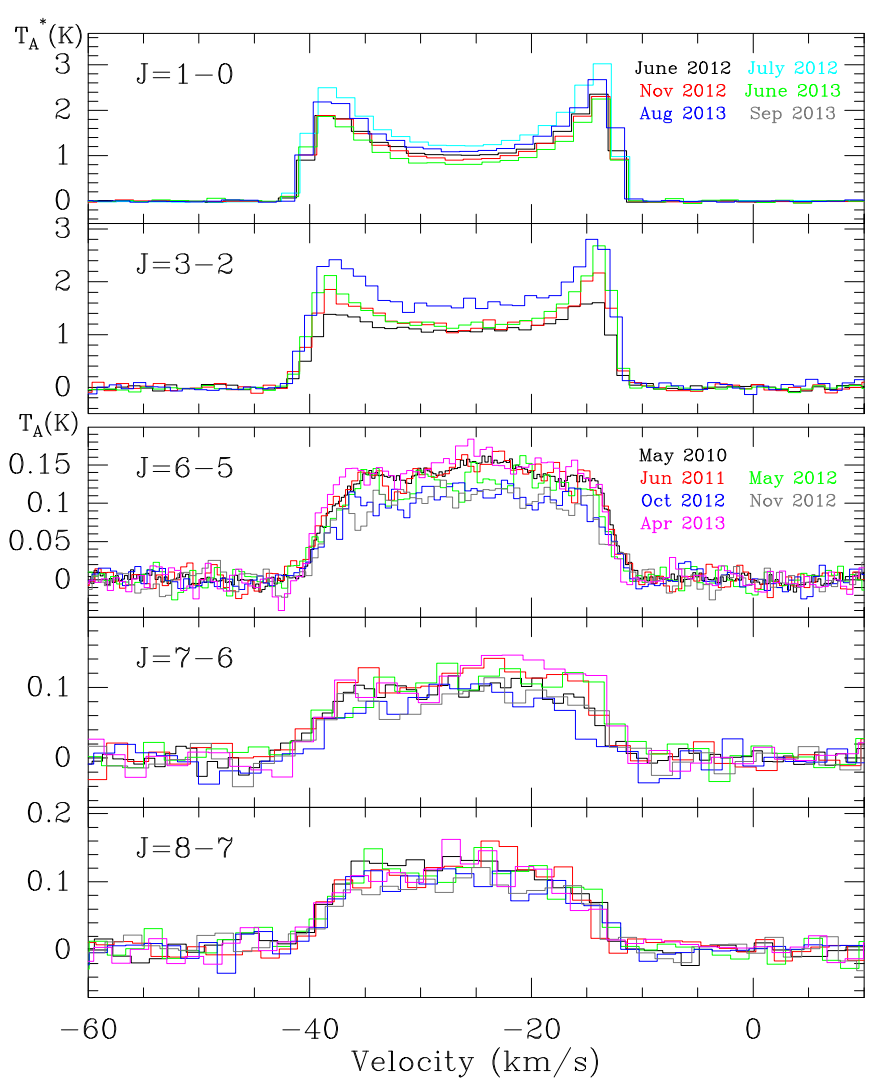

Figure 3. HNC lines observed with the IRAM $30 \mathrm{~m}$ telescope $(J=1-0,3-2)$ and with HIFI $(J=6-5,7-6,8-7)$. The different colors correspond to the observing dates with both instruments. Intensity scale is antenna temperature in $\mathrm{K}$ and the abscissa is velocity in $\mathrm{km} \mathrm{s}^{-1}$

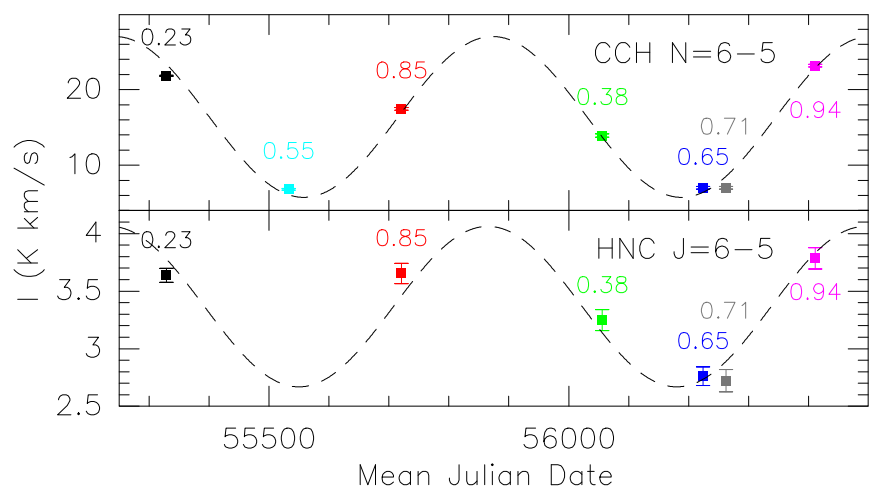

Figure 4. $\mathrm{CCH}$ (top panel) and $\mathrm{HNC}$ (bottom panel) $N / J=6-5$ integrated line intensity for each epoch using the same color code as in Figures 2 and 3. Error bars correspond to the $1 \sigma$ noise rms of the spectra. The dashed sine curve (period of 630 days) corresponds to the stellar light curve reported by Menten et al. (2012). The stellar phase is indicated for each observing date.

distance from the star and is distant from P. The magnitude of this effect on the RT depends on the period of the star, the size of the envelope, the molecular abundance profile, the local density, and the radiative transitions considered. Hence, solving the RT problem requires to couple statistical equilibrium equations with the stellar light flux and the molecular emission arising from all points of the envelope, each point seeing the stellar radiation with a different phase.

The role of infrared pumping of rotational lines has been discussed many times in the literature. Agúndez \& Cernicharo (2006) have shown that including it for $\mathrm{H}_{2} \mathrm{O}$ decreases the abundance of this molecule in IRC +10216 by a factor 10 as 

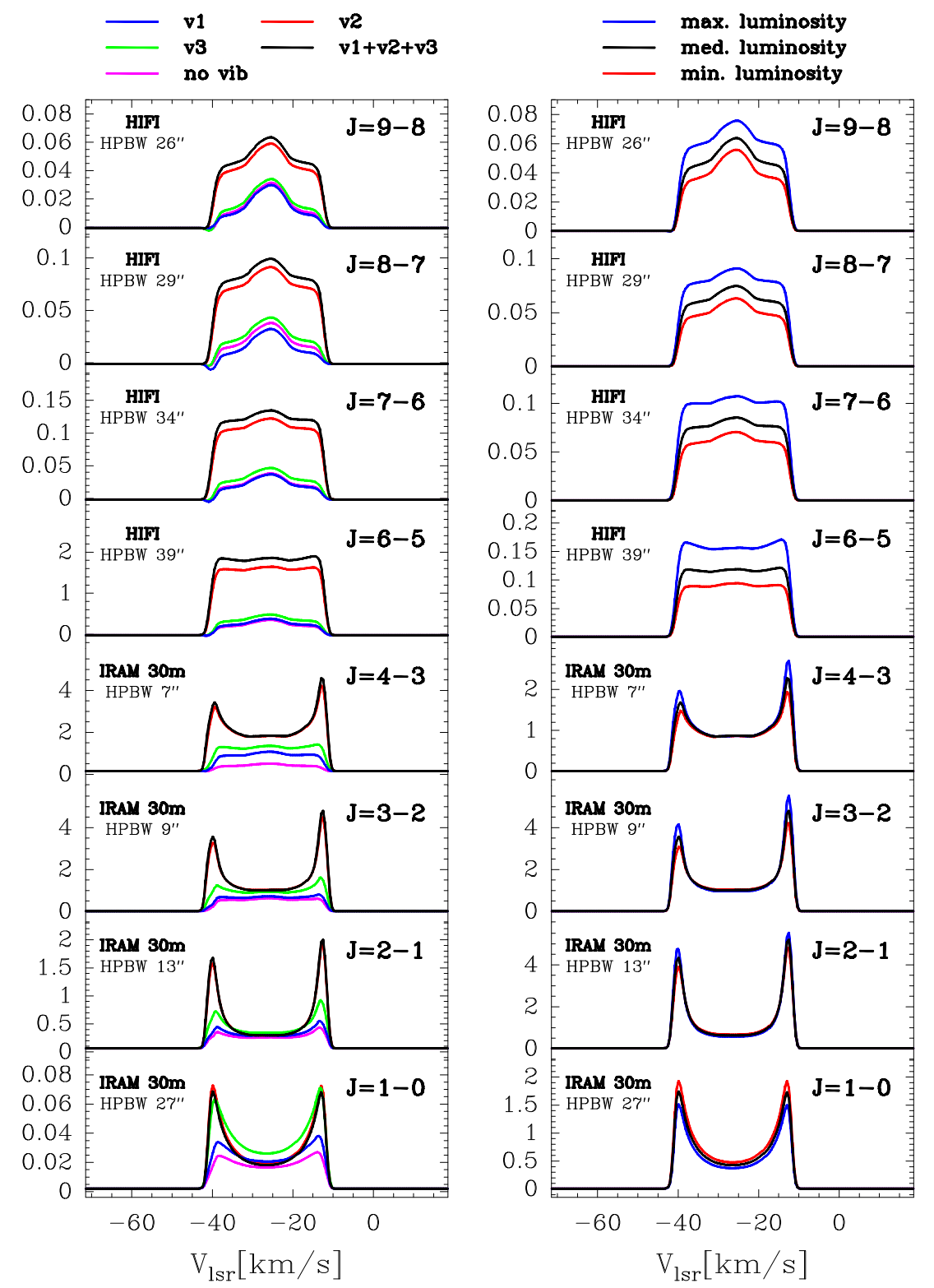

Figure 5. Left panel: effect of the infrared pumping on the population of the rotational levels of HNC for transitions from $J=1-0$ up to $J=10-9$. The different colors show the emerging spectra when each one of the vibrational modes of HNC is included in the infrared (as indicated above the top panel). The parameters adopted for the model are those of Daniel et al. (2012) and Cernicharo et al. (2013). The emerging profiles have been computed for different telescopes as indicated in each panel. The stellar phase was assumed to be 0.3. Right panel: emerging HNC profiles for three different stellar phases (as indicated above the top panel), including infrared pumping through all vibrational modes. Intensity scale is antenna temperature in $\mathrm{K}$ and abscissa is velocity in $\mathrm{km} \mathrm{s}^{-1}$ for both panels.

compared to a case in which only collisional excitation is considered. In triatomic molecules such as $\mathrm{CCH}$ and $\mathrm{HNC}$ infrared pumping has been considered by de Beck et al. (2012) and Daniel et al. (2012). CCH has a peculiarity when compared with HNC. The $v_{2}^{1}+2 v_{3}$ and $v_{1}+v_{2}^{1}$ vibronic modes of $\mathrm{CCH}$ are strongly coupled to the two lower A' components of the first ${ }^{2} \Pi$ electronic excited state. The two A" upper components of this state are strongly coupled to the ground vibrational levels $5 v_{2}^{1}+v_{3}$ and $9 v_{2}^{1}+v_{3}$. Hence, $\mathrm{CCH}$ has a complex infrared spectrum below 12,000 $\mathrm{cm}^{-1}$ (see, e.g., Carrick et al. 1983; Curl et al. 1985; Sharp-Williams et al. 2011), which is difficult to model as $17 \Pi$ vibronic states are coupled at some level, producing strong transitions to overtones and combination bands through infrared pumping (Tarroni \& Carter 2003, 2004). Daniel et al. (2012) have considered the effect of including or not the different vibrational modes of HNC in the RT modeling. The bending mode, $v_{2}$, at $21 \mu \mathrm{m}$ could be mainly populated from photons arising in the dust formation zone while the stretching modes and their combination bands with the bending mode will be populated through dust and stellar photons between 2 and $5 \mu \mathrm{m}$.

In order to quantify the role of stellar light variations, we have developed a RT model for HNC (Daniel et al. 2012) with the abundance profile for this molecule in IRC+19216 derived recently from ALMA data by Cernicharo et al. (2013). The results are shown on Figure 5. The left panel shows the relative influence of each vibrational mode on the emerging line intensities and profiles for stellar phase $\phi=0.3$. The $\nu_{2}$ bending mode at $21 \mu \mathrm{m}$ clearly dominates in all observed rotational lines. For a molecule having a large abundance in the innermost region of the CSE, which is not the case for HNC, pumping through the stretching modes will be also very important 
(HCN, for example). The right panel shows the intensities and profiles for different IR flux levels (i.e., stellar phases). The predicted low- $J$ lines show little change with the stellar phase, whereas high- $J$ lines show a strong intensity modulation. In spite of the simplicity of our model, which does not include time lags across the CSE (which could be important for large CSEs and for short period stars), the predicted line profiles and intensities reproduce well the observations shown in Figures 2 and 3. We also see that all $J / N$ lines behave differently: the $J=1-0$ line on Figure 5 (right) is brighter at minimum IR intensity, whereas the lines with high $J s$ are dimmer. Indeed, IR pumping tends to populate the high-energy $J$ levels at the expense of the lowest $J=0,1$, and 2 levels. This is actually observed for both $\mathrm{CCH} N=1-0$ and HNC $J=1-0$ in Figures 2 and 3, which show that the light blue profile, corresponding to the minimum of IR flux (2012 July, see Figure 4), is brighter than all other stellar phases.

The question of different time lags between radiatively connected points of the envelope can partly be overcome when the velocity gradient is large with respect to turbulent velocity, which is the case for radii larger than $20 R_{*}$. A large velocity gradient decouples radiatively all, but the closest points in the envelope, drastically reducing the complexity of the RT models. As molecular radiation plays in this case only a local role we can solve those equations independently at every radius $r$. The stellar radiation field will be seen at distance $r$ from the star with a time lag $r / c$. The effect on the emerging line profiles will depend strongly on the molecular line opacities. Optically thick lines arising from the whole envelope as the low- $J$ lines of $\mathrm{CO}, \mathrm{HCN}$, and $\mathrm{SiC}_{2}$ will be less sensitive to IR pumping and show little fluctuation. However, the emerging high- $J$ lines, which are very sensitive to IR pumping and arise from smaller size regions, will also show a modulation with the stellar phase.

Finally, the blue and red horns of the cusped lines, that arise from the front and rear caps of the expanding envelope, respectively, should exhibit a differential phase delay in their intensity variations, upon reaching the observer, that depends on the size of the envelope. This delay may be used to constrain the distance of CW Leo, which is still poorly known (Menten et al. 2012). In the cases of CCH and HNC, which are mainly constrained within a couple of thin spherical shells of radius $\simeq 14^{\prime \prime}-20^{\prime \prime}$ (corresponding to $3-5 \times 10^{16} \mathrm{~cm}$ if the actual distance is $130 \mathrm{pc}$ ), a delay of 20-30 days could be expected. Unfortunately, although several attempts were made with the IRAM $30 \mathrm{~m}$ telescope, at one week intervals, to observe the lowest variable line of $\mathrm{CCH}, N=4-3$, poor weather prevented us to obtain reliable data.

In conclusion, the derivation of the physical parameters of variable star envelopes, from their submillimeter and far-IR molecular emissions, is not as straightforward as previously assumed. The intensities of submillimeter molecular lines of AGB star envelopes cannot be considered as reliable flux calibrators. Even the far-IR continuum emission from those envelopes may change significantly with the stellar phase (Groenewegen et al. 2012). Nevertheless, the low- $J$ lines of $\mathrm{CO}$, which have been used to derive the mass-loss rate of AGB stars, are not significantly affected by their periodical luminosity variations. The interpretation of submillimeter and far-IR line observations of CSEs requires to elaborate RT models that include the IR flux variations and IR pumping throughout the envelope. Resulting changes in the dust and gas temperatures in the innermost layers of the CSE have also to be implemented. Each molecule in this game has its own peculiarities (abundance profile, frequency, and intensities of the vibrational modes). The spatial extent will also affect the balance between collisional and IR pumping. Moreover, these effects could be different for each isotopologue of a given molecule due to the different opacities of their rotational and ro-vibrational lines.

J.C., M.A., G.Q.L., and L.V.P. thank the Spanish MICINN for funding under grants AYA2009-07304, AYA2012-32032, CSD2009-00038, and ERC under ERC-2013-SyG, G.A. 610256 NANOCOSMOS. L.D. acknowledges financial support from the Fund for Scientific Research-Flanders (FWO). This work was based on observations carried out with the IRAM $30 \mathrm{~m}$ telescope. IRAM is supported by INSU/CNRS (France), MPG (Germany) and IGN (Spain).

\section{REFERENCES}

Agúndez, M., \& Cernicharo, J. 2006, ApJ, 650, 374

Agúndez, M., Cernicharo, J., Decin, L., et al. 2014, ApJL, 790, L27

Agúndez, M., Cernicharo, J., Waters, L. B.F. M., et al. 2011, A\&A, 533, L6

Carrick, P. G., Merer, A. J., \& Curl, R. F., Jr. 1983, JChPh, 78, 3652

Cernicharo, J., Agúndez, M., Kahane, C., et al. 2011, A\&A, 529, L3

Cernicharo, J., Barlow, M., González, E., et al. 1996, A\&A, 315, L201

Cernicharo, J., Daniel, F., Castro-Carrizo, A., et al. 2013, ApJL, 778, L25

Cernicharo, J., Decin, L., Barlow, M., et al. 2010a, A\&A, 518, L136

Cernicharo, J., Guélin, M., \& Kahane, C. 2000, A\&AS, 142, 181

Cernicharo, J., Waters, L. B.F. M., Decin, L., et al. 2010b, A\&A, 521, L8

Curl, R. F., Jr., Carrick, P. G., \& Merer, A. J. 1985, JChPh, 82, 3479

Daniel, F., Agúndez, M., Cernicharo, J., et al. 2012, A\&A, 542, A37

de Beck, E., Lombaert, R., Agúndez, M., et al. 2012, A\&A, 539, A108

Decin, L., Cernicharo, J., Barlow, M., et al. 2010, A\&A, 518, L143

de Graauw, T., Helmich, F. P., Phillips, T. G., et al. 2010, A\&A, 518, L6

Deguchi, S., \& Uyemura, M. 1984, ApJ, 285, 153

Fonfría, J. P., Cernicharo, J., Richter, M. J., \& Lacy, J. H. 2008, ApJ, 673,445

Groenewegen, M. A.T., Barlow, M., Blommaert, J. A.D. L., et al. 2012, A\&A, 543, L8

Menten, K. M., Reid, M. J., Kaminski, T., \& Claussen, M. J. 2012, A\&A, 543, A73, (NatCo, 5, 3054)

Müller, H. S.P., Cernicharo, J., Agúndez, M., et al. 2012, JMoSp, 271, 50

Nakashima, J., \& Deguchi, S. 2007, ApJ, 669, 556

Pardo, J. R., Alcolea, J., Bujarrabal, V., et al. 2004, A\&A, 424, 145

Pilbratt, G. L., Riedinger, J. R., Passvogel, T., et al. 2010, A\&A, 518, L1

Sharp-Williams, E. N., Roberts, M. A., \& Nesbitt, D. J. 2011, JChPh, 134,064314

Tarroni, R., \& Carter, S. 2003, JChPh, 119, 12878

Tarroni, R., \& Carter, S. 2004, MolPh, 102, 2167 